\title{
Adaptive genomic evolution of opsins reveals that early mammals flourished in nocturnal environments
}

\author{
Rui Borges ${ }^{1,2}$, Warren E. Johnson ${ }^{3}$, Stephen J. O'Brien ${ }^{4,5}$, Cidália Gomes ${ }^{1,6}$, Christopher P. Heesy ${ }^{7}$ \\ and Agostinho Antunes ${ }^{1,2^{*}}$
}

\begin{abstract}
Background: Based on evolutionary patterns of the vertebrate eye, Walls (1942) hypothesized that early placental mammals evolved primarily in nocturnal habitats. However, not only Eutheria, but all mammals show photic characteristics (i.e. dichromatic vision, rod-dominated retina) suggestive of a scotopic eye design.

Results: Here, we used integrative comparative genomic and phylogenetic methodologies employing the photoreceptive opsin gene family in 154 mammals to test the likelihood of a nocturnal period in the emergence of all mammals. We showed that mammals possess genomic patterns concordant with a nocturnal ancestry. The loss of the $\mathrm{RH} 2$, $V A, P A R A, P A R I E$ and OPN4x opsins in all mammals led us to advance a probable and most-parsimonious hypothesis of a global nocturnal bottleneck that explains the loss of these genes in the emerging lineage $(>>215.5$ million years ago). In addition, ancestral character reconstruction analyses provided strong evidence that ancestral mammals possessed a nocturnal lifestyle, ultra-violet-sensitive vision, low visual acuity and low orbit convergence (i.e. panoramic vision).
\end{abstract}

Conclusions: Overall, this study provides insight into the evolutionary history of the mammalian eye while discussing important ecological aspects of the photic paleo-environments ancestral mammals have occupied.

Keywords: Nocturnal bottleneck, Mammals, Opsins, Nocturnal lifestyle, Ultra-violet sensitive vision, Panoramic vision, Visual acuity

\section{Background}

Walls, in 1942, first recognized that placental mammals have anatomical eye and retinal characteristics that are suitable to an ancestral nocturnal lifestyle and introduced the nocturnal bottleneck hypothesis: "We can be sure that at an early period in a placentalian evolution, the only placentals on earth were so thoroughly nocturnal..." [1]. Supported by very detailed comparativeanatomy studies in mammals and other vertebrates, Walls demonstrated that eutherians possess markedly spherical eyes and do not have oil droplets - retinal components which facilitate colour discrimination [2].

\footnotetext{
* Correspondence: aantunes@ciimar.up.pt

${ }^{1}$ CIIMAR/CIMAR, Interdisciplinary Centre of Marine and Environmental Research, University of Porto, Terminal de Cruzeiros do Porto de Leixões, Av. General Norton de Matos, s/n, 4450-208, Porto, Portugal

2Department of Biology, Faculty of Sciences, University of Porto, Rua do Campo Alegre, 4169-007 Porto, Portugal

Full list of author information is available at the end of the article
}

However, not only Eutheria, but all mammals present photic-related structures that suggest past adaptation to nocturnal environments. For example, mammals have fewer photoreception organs, and therefore rely on the eye as the sole photoreceptive organ $[3,4]$. In contrast with diurnal Tetrapoda, which are mostly tetrachromatic (possess four types of cone-cells involved in colour vision), mammals are mostly dichromatic and thus less able to discriminate colours. In addition, mammals have a clear preponderance of rod cells in the retina $[5,6]$, which perform notably well in low-light conditions [5]. Furthermore, the mammalian eye design is in some aspects indicative of being scotopic adapted (i.e. low-light adapted) [7]. Mammals present the highest convergence of orbital disposition (facing in a similar direction) among amniotes and thus the largest binocular visual fields [8], which indicates better perception of object texture, greater discrimination of contrast, and improved 
light sensitivity $[8,9]$. Mammals also possess higher relative corneal diameters [7], presumably improving visual sensitivity under scotopic light conditions [1], and smaller absolute axial eye length, particular when compared with birds [10]. Higher axial eye length has been associated with higher visual acuity and thus with photopic-adapted (i.e. well-lit adapted) eyes [1, 8].

At the molecular level, mammalian photoreception is mediated by opsins (Table 1), which are heptatransmembrane proteins involved in the detection of photic stimuli $[11,12]$. Compared with other vertebrates, mammals have lost two visual opsins: it was reported that $\mathrm{RH} 2$ is missing in all mammals, while therians have lost the OPN1sw2 and monotremes the OPN1sw1 [5]; furthermore, one representative of the melanopsin subfamily $(O P N 4 x)$ is absent in mammals $[13,14]$.

While a nocturnal ancestry appears possible for all mammals, and not only for placentals as Walls firstly assumed [1], a proper phylogenetic approach conjugating ecological, macro-evolutionary, and molecular photic adaptations of mammals still needs to be implemented. Given the central role of opsins mediating photic-related responses, they are ideal genes to retrace the evolution of the mammalian eye. Here, we used integrative comparative genomics and evolutionary methodologies employing the opsin gene family across $\sim 154$ mammals to test the likelihood of a global nocturnal bottleneck.

\section{Results}

\section{Synteny analysis of tetrapod opsins}

tblastn searches in mammalian and non-mammalian tetrapod genomes were performed to assess opsins presence and absence $[15,16]$. We confirmed that amphibians, reptiles and birds have 16, 17 and 15 opsin genes, respectively, while only 10 opsin genes were identified in mammals (RH1, OPN1sw1, OPN1sw2, OPNlw, OPN3,
TMT, OPN5, RGR, RRH and OPN4m). Further exploration of the absent mammalian opsins among the tetrapod genomes (Fig. 1) showed the existence of a conserved synteny in the genomic regions where $R H 2$, $V A, P A R A, P A R I E$ and OPN4X opsins were located, suggesting that these opsins were lost in mammals. The same could not be advanced to the PIN and TMT2 opsins due to the inconsistent syntenic patterns of these genomic regions (Fig. 1). According to the syntenic analysis, mammals differ in their repertoire of opsins: monotremes were the only lineage with OPN1sw2, but not OPN1sw1, which was only present in therians; TMT was only present in marsupials. We also observed that $R G R$ was absent in marsupials and, OPN3 was absent in monotremes; however, due to evidence of genomic rearrangements in these regions, these absences could not be syntenic validated as lineage-specific losses (Fig. 1).

\section{Site- and branch-selection in mammalian opsins}

We implemented site-specific selection tests based on the $\omega$-ratio (the ratio between the non-synonymous and synonymous rate of substitution, $\omega=d N / d S$ ) to assess the selective pressures acting on the mammalian opsins $[17,18]$. OPN1sw1, OPN4m, OPN3 and RRH showed evidence of site-specific positive selection, while $R H 1$ showed evidence of site-specific negative selection (Additional file 1: Table S1). In addition, the OPN1sw1conopsin 50I, 93P, $100 \mathrm{~N}, 108 \mathrm{H}, 314 \mathrm{Q}$ and 334 T residues (human amino acid residues, based on bovine rhodopsin numbering) were positively selected (Bayes empirical Bayes >0.75; Fig. 2). Site 93 has been shown experimentally to be involved in the OPN1sw1 spectral tuning $[19,20]$. Further analysis demonstrated that the amino acid composition at site 93 segregates with the nocturnal/diurnal activity patterns of mammals (bar plot of Fig. 2), as T, S, A and $\mathrm{M}$ amino acids were

Table 1 Tetrapoda opsins and their functions. Subfamilies characterization according to [5, 12, 78] and [4]

\begin{tabular}{|c|c|c|}
\hline Subfamily & Opsins & Functions \\
\hline Visual opsins & $\begin{array}{l}\text { RH1 } \\
\text { RH2 } \\
\text { OPN1sw1 } \\
\text { OPN1sw2 } \\
\text { OPN1/W }\end{array}$ & $\begin{array}{l}\text { Rhodopsin mediates vision in dim-light whereas conopsins are responsible for colour vision. OPN1/W } \\
\text { is sensitive to red-green or long-wavelengths, } R H 2 \text { to green or middle wavelengths and two short-wave } \\
\text { conopsins (OPN1sw2 and OPN1sw1) respond to blue-violet or violet-ultraviolet wavelengths, respectively. }\end{array}$ \\
\hline Non-visual opsins (sensu stricto) & $\begin{array}{l}\text { OPN3 } \\
\text { TMT } \\
\text { TMT2 }\end{array}$ & $\begin{array}{l}\text { TMT (teleost-multiple-tissue) are expressed in the liver, kidney and heart as well as eye and brain. } \\
\text { Phylogenetic analysis reveals that it clades with OPN3, which also exhibits a multiple patterns of } \\
\text { tissue expression. }\end{array}$ \\
\hline Pineal opsins & $\begin{array}{l}\text { PARA } \\
\text { PARIE } \\
\text { PIN } \\
\text { VA }\end{array}$ & $\begin{array}{l}\text { Multiple opsins (PARA, PARIE, PIN) have been isolated from the parapineal complex. VA opsin is also } \\
\text { expressed in the retina and was shown that forms a functional photopigment sensitive in the } \\
460-480 \mathrm{~nm} \text { range. }\end{array}$ \\
\hline Photoisomerases and Neuropsins & $\begin{array}{l}\text { OPN5 } \\
\text { RGR } \\
\text { RRH }\end{array}$ & $\begin{array}{l}\text { RGR and } R R H \text { have a probable role as all-trans retinal photoisomerases. OPN5 shows an absorption } \\
\text { maximum at } 380 \mathrm{~nm} \text { and is thus UV-sensitive. }\end{array}$ \\
\hline Melanopsins & $\begin{array}{l}\text { OPN4X } \\
\text { OPN4m }\end{array}$ & Melanopsins are involved in circadian rhythm regulation and pupillary light reflexes. \\
\hline
\end{tabular}




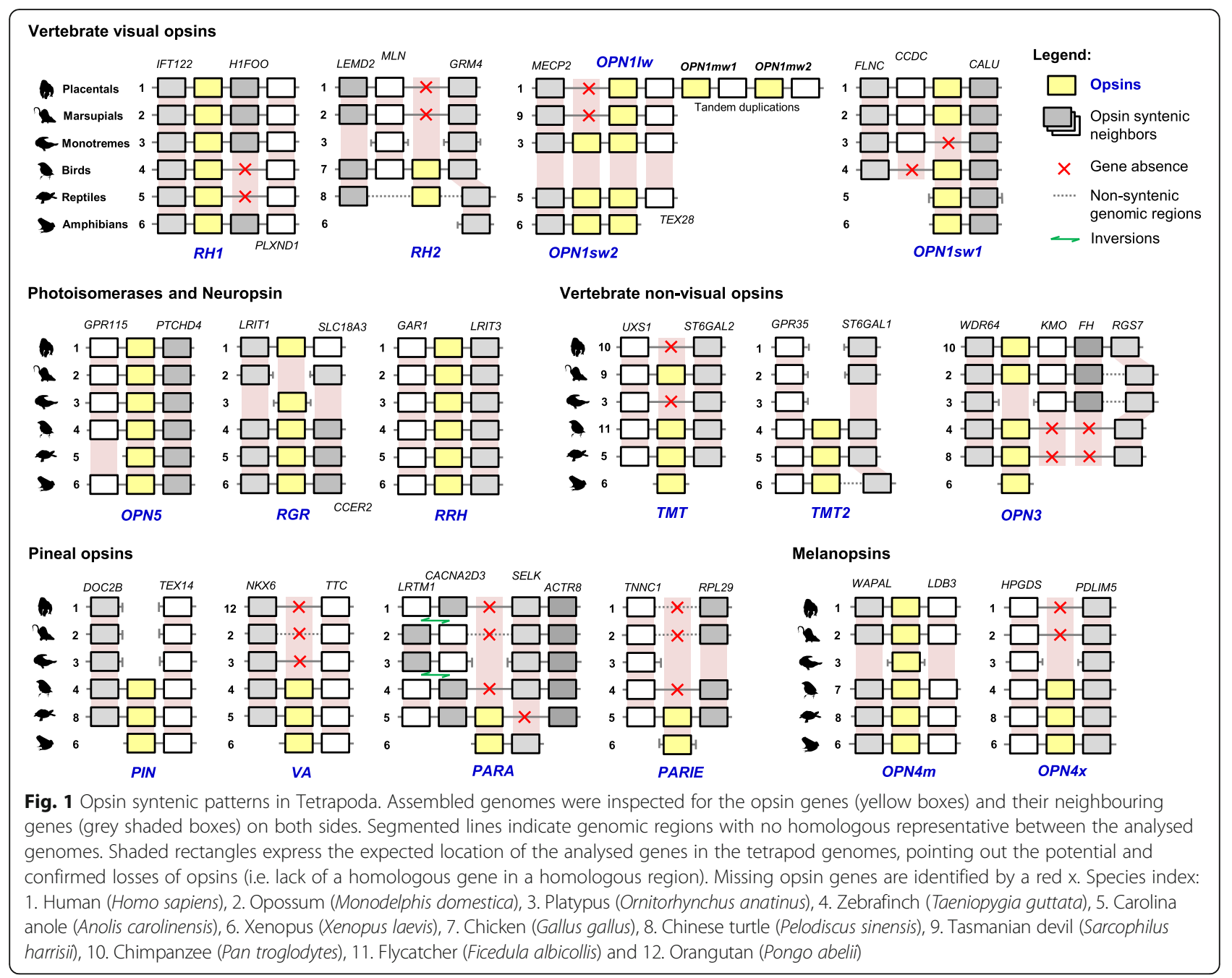

mostly associated with nocturnal lineages, while P, V and $\mathrm{C}$ were with diurnal.

To assess the prevailing selective pressures acting at the branch level in the mammalian opsins, we performed branch-specific selection analysis. The branch selection models were implemented in two phases: computing the free-ratios model to calculate the opsins adaptive trees ( $\omega$-trees) and test ing the one-ratio vs. two-ratio hypothesis to validate lineage-specific adaptive events [21]. The species-specific evolutionary rates ( $\omega$-lineages), corresponding to the sum of the root-to-tip $\omega$-branches in each of the opsin $\omega$-trees (i.e. a $\omega$-tree linearization; Additional file 2: Table S2) were analysed and suggested outlier tendencies among Monotremata, Marsupialia, Carnivora, Chriroptera, Artiodactyla and Primata (Additional file 3: Figure S1). The root-to-tip procedure was particularly effective recovering $\omega$-ratio variations in the terminal branches and clades of the mammalian tree: we predict that this pattern is due to the treelinearization, which might dilute $\omega$-lineages tendencies in the internal branches. Thus, we proceed with these results by focusing on the most-recent adaptations of mammals. Further analyses of these lineages were carried out by applying the one-ratio vs. two-ratios branch-specific test and significant results were obtained ( $p$-value $<0.002$; Bonferroni corrected for 24 tests) in the terminal lineages of platypus (Ornitorhynchus anatinus, RRH, Likelihood ratio test $(\mathrm{LRT})=11.849$; OPN4m, LRT $=19.186)$, bearded seal (Erignathus barbatus, RH1, LRT $=10.312$ ) and Sowerby's beaked whale (Mesoplodon bidens, RH1, LRT = 29.651) (Additional file 4: Table S3).

\section{Ancestral character reconstructions}

In order to retrace the photic evolution of the mammalian eye, ancestral reconstructions were carried out in the emerging nodes of mammals, monotremes, therians, marsupials and placentals for four photic-related characters [activity pattern, violet/ultra-violet sensitive (VS/ UVS) vision, orbit convergence and visual acuity]. 


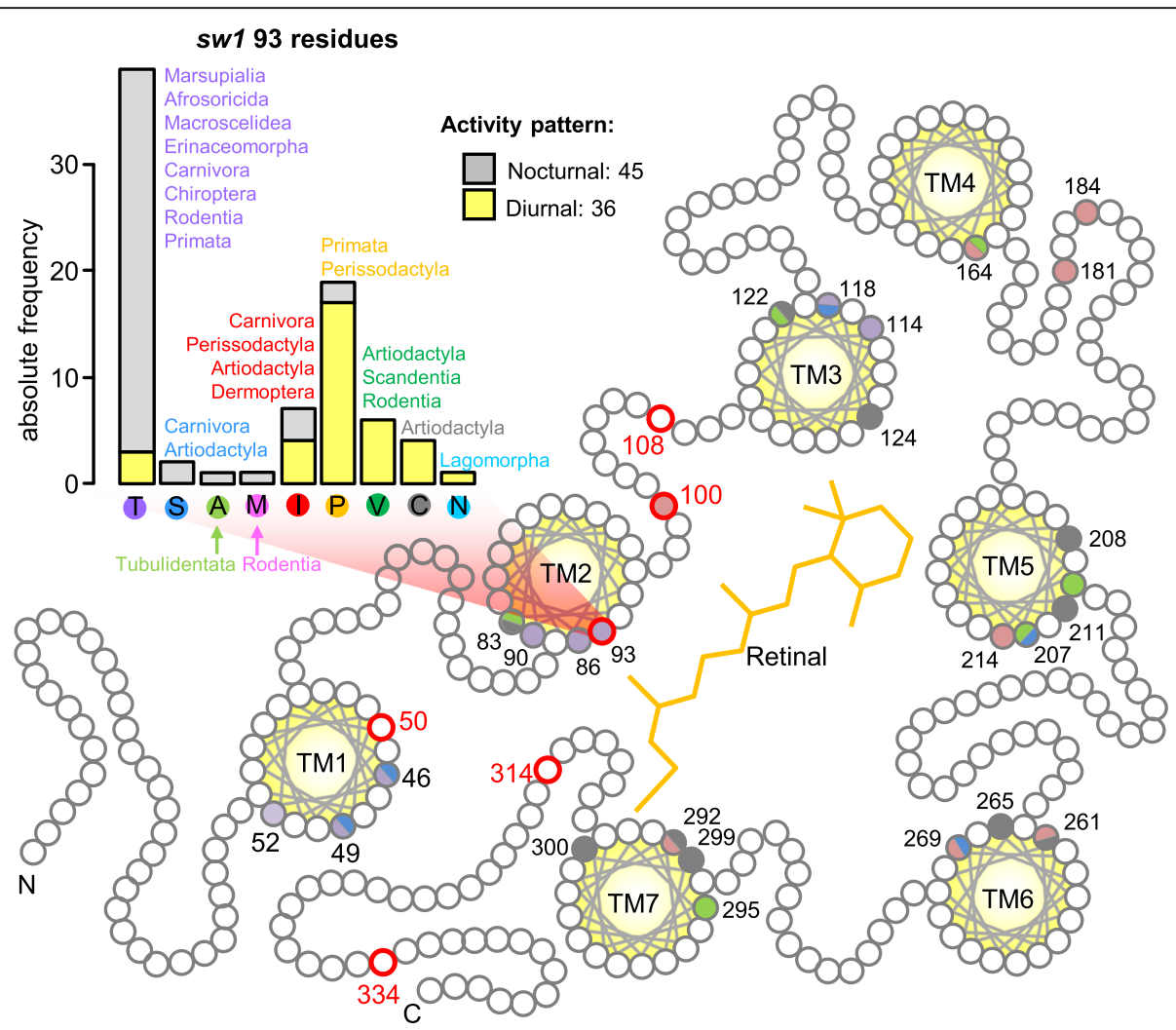

Fig. 2 Selective signatures in the mammalian OPN1sw1 opsin. Schematic view of the bovine rhodopsin highlighting the visual opsins spectral tuning sites ([19, 20]): OPN1/W (red), RH2 (green), OPN1sw2 (blue), OPN1sw1 (violet) and RH1 (black). Shared spectral tuning sites are indicated by shared colours. Residues highlighted in red experienced positive selection in mammals. The bar plot depicts the amino acid composition of the OPN1sw1 93 spectral tuning site accounting for the nocturnal and diurnal species and the eutherian orders (or infraclass in the case of marsupials) in which these amino acids were found

Ancestral reconstructions for the mammalian nocturnal, cathemeral and diurnal activity patterns and VS/ UVS vision were implemented using the RH1 and OPN1sw1 $\omega$-trees, respectively (Additional file 2: Table S2). Previous studies validated the use of these opsins as genetic markers in making ancestral inferences, such as the RH1 mediating the phototransduction in low-light levels, which has been related with the mammalian activity pattern evolution [22-26], whereas sequence variations in the OPN1sw1 determine spectral tuning changes in the violet/ultra-violet range [27-30]. The allrates-different time-continuous Markov model adequately fitted the evolution of activity pattern $(\mathrm{LRT}=$ 9.743, $p$-value $=0.002)$ and VS/UVS vision $(\mathrm{LRT}=8.050$, $p$-value $=0.004$ in mammals. The maximum likelihood ancestral inferences suggested a nocturnal lifestyle (i.e. nocturnal state with higher probability) in the ancestral nodes of mammals, monotremes, therians, marsupials and placentals $(0.743,0.859,0.791,0.482$ and 0.794 respectively; pie charts in Fig. 3 and Additional file 5: Figure S2). Ancestral reconstructions of VS/UVS vision strongly supported (with probability 1.000) the UVS condition in the ancestral therians, marsupials and placentals (sw1 sensitivity in Fig. 3; Additional file 5: Figure S2). OPN1sw1 is not present in monotremes. Therefore, the VS/UVS inference in the ancestral node of mammals could not be determined.

The ancestral character reconstructions were also performed for the orbital convergence and visual acuity. Orbit convergence (degrees, ${ }^{\circ}$ ) measures the orientation of the orbit bone relative to the midsagittal plane $[8,9,31]$; visual acuity (cycle per degree, cpd) is a measure of the resolution capacity of the visual system [1]. Both orbit convergence and visual acuity were primarily tested for phylogenetic autocorrelation and were significantly dependent on the opsin $\omega$-trees (Moran's I test, $p$-values $<0.003$, Bonferroni corrected for 16 comparisons; Additional file 6: Table S4), demonstrating that opsin genes are appropriate genetic markers to perform ancestral inferences for these characters. The maximum likelihood inferences, obtained employing the Brownian motion model in the opsin $\omega$-trees, indicated low orbit convergence $\left(<42^{\circ}\right.$, i.e. panoramic vision; the orbit orientation can be used as a surrogate variable for the degree of panoramic/binocular vision [9]) and a low visual acuity (< $7 \mathrm{cpd}$ ) in all the studied nodes (mammals, monotremes, 


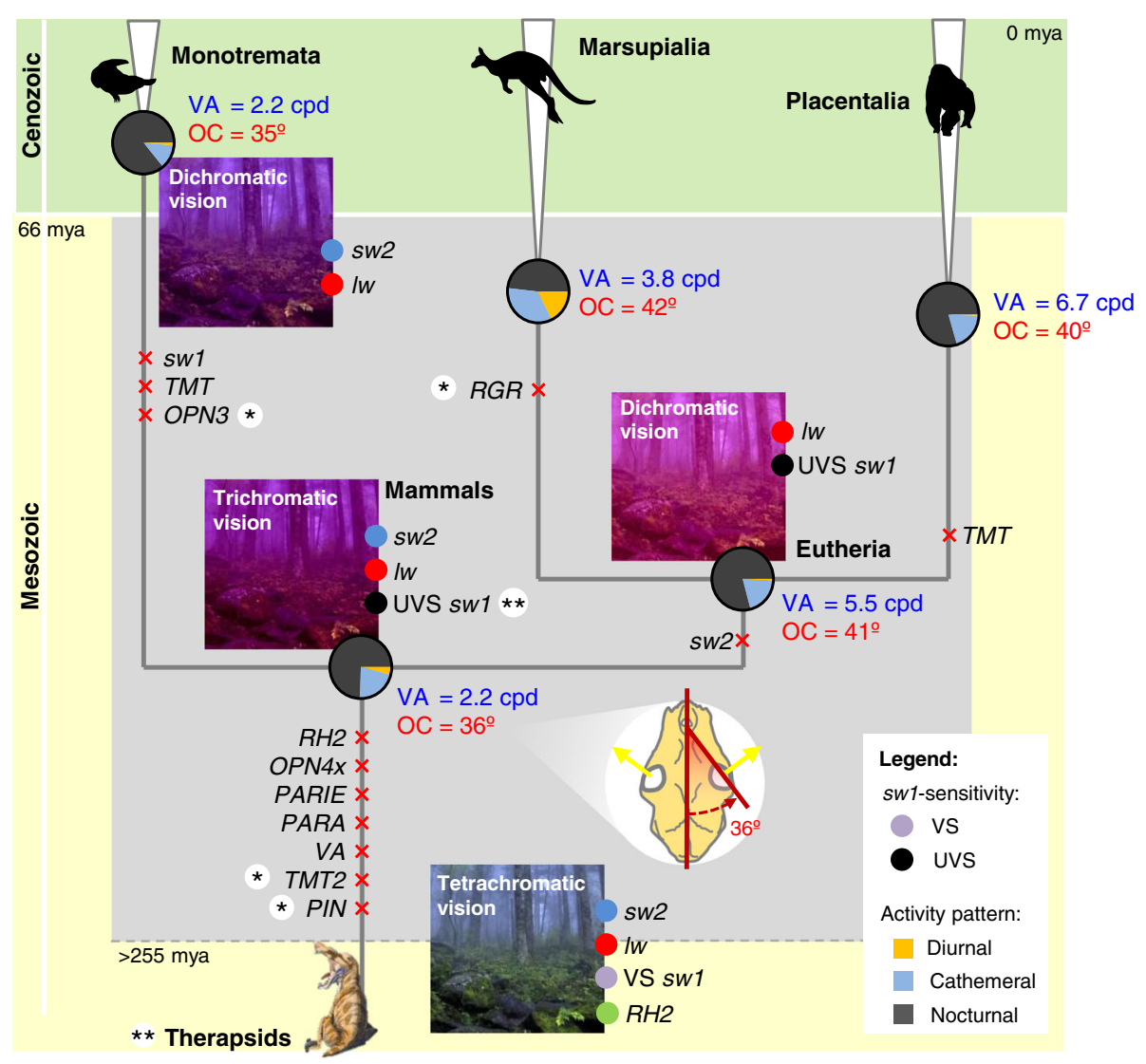

Fig. 3 Evolution of opsins and photic-related characters in mammals. Schematic view of the global nocturnal bottleneck hypothesis, linking both the results from the synteny and the ancestral reconstruction analyses: a nocturnal period (represented in grey) is assumed to affect both the mammalian ancestor and the emerging lineages. Global and lineage-specific losses of opsins are indicated with a red cross; opsin losses that were not supported by a consistent synteny are marked with an asterisk (*). Ancestral reconstructions of the activity pattern are represented in pie charts, each slice representing the probability of each state (nocturnal, diurnal and cathemeral). Ancestral reconstructions of the violet and ultra-violet sensitive (VS and UVS) vision are represented by a violet or a black circle, respectively, near the OPN1SW1 opsin (sw 1 was used to perform the inferences). Uncertainties about the opsin presence/absence are marked with a double asterisk $\left(*^{*}\right)$ : UVS vision in the mammalian ancestor node is only hypothetical (it could not be determined because the sw1 is not present in monotremes); the therapsid opsin profile is not known since genetic data is not available for therapsids. The potential vision of ancestral mammals was inferred considering both the conopsin content of each node and their spectral sensitivities [blue for OPN1sw2 (sw2), red for OPN1/W (IW) and saturated pink for UV sw1]. Ancestral inferences of the orbit convergence (OC, degrees) and visual acuity (VA, cycles per degree) are indicated in the corresponding nodes. A reconstruction of a skull with an orbital convergence angle of $46^{\circ}$ (which correspond to the inferred angle in the node of mammals) is included. The Shennongjia virgin forest (https://commons.wikimedia.org/wiki/File:Shennongjia_virgin_forest.jpg) and the Pristerognathus (https://commons.wikime dia.org/wiki/File:Pristeroognathus_DB.jpg) are licensed under the Attribution-ShareAlike 3.0 Unported license (the license terms can be found on the following link: https://creativecommons.org/licenses/by-sa/3.0/)

eutherians, marsupials and placentals; Additional file 5: Figure S2, Additional file 6: Table S4 and Fig. 3). The inferred phenotypes are similar to the orbit disposition and visual acuity observed in the nocturnal brown rat $\left(32^{\circ}\right.$ and 1.6 cpd, respectively) but contrast with humans, which have high orbit convergence and visual acuity $\left(79.3^{\circ}\right.$ and $64 \mathrm{cpd}$, respectively) [32-34]. Furthermore, we found a significant association between the orbit convergence and the nocturnal/diurnal activity patterns of extant mammals, suggesting that nocturnal mammals generally have divergent orbits while diurnal mammals possess convergent orbits ( $p$-value $=0.0076$, Wilcoxon test, Additional file 7: Figure S3).
Since branch-site tests of positive selection can retrieve misleading results [26], our study considered both branch- and site-specific selection tests (the branch-site tests have been then discarded from the analyses). Moreover, to increase the confidence of our results: $(i)$ we have considered only the sites under positive selection that have been reported as having a role in spectral tuning (in our case, the site 93 of the OPN1sw1); (ii) the branch-selection tests were Bonferroni corrected avoiding false positives; (iii) the ancestral inferences for the discrete characters (activity pattern and UVS/VS vision) were only used with the opsin $\omega$-trees with known role 
in these processes (i.e. $R H 1$ and $O P N 1 s w 1$, respectively); and $(i v)$ the inferences for the continuous characters (visual acuity and orbit convergence) were firstly tested for phylogenetic signal.

\section{Discussion}

\section{Opsin gene content in ancestral mammals}

Based on genomic analyses, we detected that the RH2, VA, PARA, PARIE and OPN4x (and possibly PIN and TMT2) opsins were absent in mammals, suggesting that ancestral mammals evolved with a reduced number of opsins. The extensive loss of opsins from the mammalian genomes indicates that early mammals should have inhabited environments where relevant photic stimuli were absent, permitting the corresponding opsins to become pseudogenized with none (or minor) effect on species fitness. A nocturnal phase (or a progressively nocturnal phase) in the early mammals would be concordant with the loss of the RH2,VA, PARA, PARIE and OPN4x opsins.

The $R H 2$ photoreceptor responds to the green range of the light spectrum [12] and its loss represented a decrease in the ability of mammals to discriminate colours. While the RH2 loss does not imply that colour vision was compromised in ancestral mammals since they most probably had a trichromatic visual system with the OPN1sw1, OPN1sw2 and OPN1lw conopsins, it is suggestive that visual acuity was being reduced, which constitutes a plausible adaptation to scotopic or mesopic environments. Moreover, the $\mathrm{RH} 2$ pseudogenization was reported in other nocturnal species, as the barn owl, indicating that the loss of the $\mathrm{RH} 2$ pigment must be common in scotopic environments [35].

The loss of the OPN4x opsin suggests that mammals have simplified their circadian responses. However, they were not compromised because the $m$-type melanopsin $(O P N 4 m)$ is still present in the mammalian retina. Melanopsins are expressed in a particular group of retinal cells localized in the ganglion cell layer, where they performed non-image forming tasks [14]. Notably, this cell layer was reported to be very reduced in the nocturnaltype retina [1] which could have potentiated the loss of the OPN4x in mammals. Nevertheless, further studies in the melanopsin gene family are still necessary to understand the consequences of losing OPN4X and maintaining OPN4m in the circadian response. The same applies to the TMT gene family, for which a possible loss was reported (TMT2): not only the TMT opsins have an undifferentiated expression (both were reported to be expressed in the eyes, brain and other internal organs of vertebrates) but also their photoreceptive roles remain, particularly for Tetrapoda, unknown [4].

We have evidence that all the pineal opsins (PARA, PARIE, VA and PIN) were lost from the mammalian genomes. Pineal opsins are expressed in the third eye of vertebrates, which is responsible for the regulation of circadian rhythms and hormone production for thermoregulation [4]. The mammalian third eye, in contrast with other vertebrates, lacks a parietal organ and has a pineal organ with secretory functions only (pineal gland) [36]. We propose that the extensive loss of pineal opsins is correlated with the simplification of the third eye in mammals due to their nocturnal emergence. In nocturnal environments, which are less energetic environments, nocturnal mammals have to develop new thermoregulation strategies to become less reliant on external sources of energy to maintain body temperature: indeed, in contrast with their ancestors, mammals are endothermic [37, 38]. Thus, we propose that the third eye, which most certainly had a role in the ectothermic response [39], partly degenerated when mammals evolved to depend on endothermic metabolism, leading to the loss of the pineal opsins. Likewise, the loss of two pineal opsins (PARA and PARIE) has also been reported in birds, which like mammals, are endothermic [35].

The syntenic analyses showed global (RH2, OPN4x, TMT2, PIN and VA and possibly PIN and TMT2 were all absent in mammals) but also lineage-specific losses of opsins among mammals (monotremes were the only lineage with OPN1sw2; OPN1sw1 and OPN3 were present only in therians; TMT was only present in marsupials; the $R G R$ gene was absent in marsupials) thus suggesting that the nocturnal period continued after the mammalian lineages diverged, affecting both the most recent common ancestor of mammals [215.5 million years ago (mya), which corresponds with the emergence of mammals [40]] and the earliest emerging lineages. Hence, we propose a mesopic-to-scotopic bottleneck: in an initial mesopic period the RH2, OPN4x, TMT2, PIN and $V A$ were lost, but OPN1sw1, OPN1sw2 and TMT were retained, and subsequently differentially segregated among monotremes, marsupials and placentals in a scotopic period. Indeed, the progressive decrease of visual acuity, expected by the initial loss of $R H 2$ in all mammals and then the loss of OPN1sw1 in monotremes and OPN1sw2 in therians, suggests that mammals went through photic environments with progressively lower luminance levels.

Recent evidence revealed that the majority of therapsids were mesopic or scotopic [41], suggesting that the occupation of nocturnal niches may have started before the divergence of therapsids and mammals. However, the same study also showed that the nocturnal activity appeared early in the synapsid history, evolving independently several times [41]. Without knowing which were the opsin syntenic patterns in therapsids, we can only advance that the beginning of the nocturnal bottleneck was before the emergence of mammals, i.e. > > 
215.5 mya. While the beginning of the nocturnal bottleneck cannot be stated precisely, the end of the nocturnal bottleneck is generally agreed to have occurred during the Cretaceous/Paleogene boundary (66 mya), in which the mass extinction of the large reptiles, provided mammals with the opportunity to occupy diurnal niches [38, 42]. In agreement, the predominant diurnal mammalian orders were shown to evolve at $<66$ mya (anthropoid primates, artiodactyls and perissodactyls evolved at 31.3, 65.4 and 57.0 mya [40], respectively).

\section{Scotopic and UVS vision in ancestral mammals}

In this study, we found evidence that $R H 1$ evolved under purifying selection and associated with the activity pattern of mammals, suggesting they had a nocturnal lifestyle for most of their evolutionary history. It is expected that a nocturnal lifestyle would rely on the role of the rhodopsin (RH1), which is expressed in the rods that are photoreceptive cells activated at low luminance levels [5, 6]. Thus, we advance that the preservation of the RH1 functions relates to the retention of the rods in the mammalian retina (which is rod-dominated) to guarantee dim-light photic responses at higher levels in the brain (e.g. increased visual sensitivity) [43]. A completely different scenario of $R H 1$ adaptive evolution was observed in birds, which evolved with evidence of sitespecific positive selection [35]. Birds, distinct from mammals, are more-highly visual, and with the exception of some specific lineages (e.g. strigiformes and apterygiformes), generally occupy diurnal niches [35]. Thus, the selective signatures on the RH1 gene appear to correlate with nocturnal/diurnal lifestyles. Recent evidence suggests that the conservative evolution of the NRL eye development gene in mammals is associated with the augment of rod photoreceptors in the mammalian retina [44]. These results provide evidence of an evo-devo mechanism for the activity pattern evolution in mammals and birds.

Evidence of diversifying selection was detected in the OPN1sw1 opsin, particularly in the 93 spectral tuning site, indicating that mammals evolved adaptive strategies that included the retuning of the OPN1sw1 sensitivity. Site 93 has been previously reported to be involved in the OPN1sw1 tuning: the P93T substitution significantly shifts the OPN1sw1 into the UVS in aye-aye primate [19], and also it is involved in synergistic effects with other sites $(46,49,52,81,86,114$ and 118) [20]. We observed that the amino acid variability at site 93 was related to the activity pattern of mammals, where the $93 \mathrm{~T}$ (which provides UVS) is mostly associated with nocturnal lineages and the 93P (which provides VS) with diurnal. The scotopic/UVS and photopic/VS associations were expected since many nocturnal mammals have lost mechanisms of ultra-violet-blocking and are ultra-violet- sensitive [38]. In addition, our findings are congruent with Hunt et al. (2009) who stated that the OPN1sw1 opsin was firstly adapted to respond to ultra-violet light and later evolved to become more sensitive to light within the violet range [5]: we could infer that ancestral mammals, including the most recent common ancestor of eutherians, placentals and marsupials (and for which we have inferred a nocturnal lifestyle) possessed UVS vision. Evolving a UVS OPN1sw1 in nocturnal environments suggests ancestral mammals would benefit from having this sensitivity while in dim-light; however, a photo-biological reason for such adaptation remains elusive. Maybe, ancestral mammals would benefit from the ultraviolet vision during the twilight periods of the day, which would provide the necessary luminance for the activation of the sw1 pigment [45].

\section{Ecology of the mammalian ancestral eye}

Ancestral character reconstructions showed that ancestral mammals (including the early monotremes, therians, marsupials and placentals) possessed low visual acuity and low orbit convergence. The inferred phenotypes are compatible with the general conformation of the extant nocturnal mammals.

Evidence of lower visual acuity in early mammals supports a scotopic-adapted retina. Indeed, in nocturnal environments it is more important to maximize the amount of light one can capture, regardless of being able to distinguish among spectral wavelengths $[7,8]$. Veilleux et al. (2014) demonstrated that lower visual acuity is associated with nocturnal species [34]; in addition, it has been shown that adaptations which enhance visual sensitivity in low-light are generally incompatible with high acuity $[1,46]$. This is partially from the differentiated number of rods (more sensitive to small quantities of light; more common in scotopic retinas) and cones (less sensitive but more accurate for detail, i.e. better performance distinguishing between different colours; more common in photopic retinas) in scotopic and photopic adapted retinas ([43, 47] and reviewed in [48]). Thus, lower visual acuity is associated with rod-dominated retinas specialized for enhancing visual sensitivity, characteristics that we assign to the ancestral mammals. Two pieces of evidence corroborate this statement: $(i)$ the preponderance of rods and (ii) the absence of the $R H 2$ opsin in extant mammals $[5,6]$.

Our analysis also revealed that early mammals had a lateral disposition of their orbits, thus suggesting the possession of panoramic vision. Presumably, nocturnal animals would benefit from a frontal disposition of the orbits (binocularity) because it maximizes the sensitivity to low-light levels by doubling the chance of registering a photon on the visual field [9]. Birds are a clear example: while most of the diurnal birds possess a 
divergent pattern of orbit disposition, the nocturnal ones (e.g. owls) have frontally placed eyes in order to increase visual sensitivity [49]. In mammals, this tendency appears to be inverted: our results showed that extant nocturnal mammals tend to have panoramic vision. It must be noted that Walls (1942) reported that binocular contrast sensitivity is only slightly more effective than monocular sensitivity in a normal visual system [1], and thus panoramic vision, while not being the optional phenotype one would expect in scotopic environments, does not necessarily imply a decreased visual sensitivity. However, if mammals could have developed strategies that increased visual sensitivity with both binocular and panoramic vision, why did early mammals evolve with a clear pattern of divergent orbits? An alternative hypothesis to explain the role of panoramic vision in ancestral mammals may be related to differences in prey/predator lifestyle. Panoramic visual fields have been associated with taxa subjected to predation (such as artiodactyls, equids and lagomorphs) and often is considered to be an advantage for identifying approaching predators $[1,46]$. A divergent configuration of the orbits would provide a wider field of vision and a broader view of the surrounding area, thus simultaneously allowing the detection of photic stimuli from different directions. In addition, it was shown that predators have generally higher visual acuity [34], which decreases the likelihood of ancestral mammals (for which we inferred low visual acuity) being highly-efficient predators. Therefore, we hypothesize that panoramic vision in ancestral mammals facilitated the identification of potential predators. This hypothesis is consistent with both the predation pressures imposed by the successful reptiles during the Mesozoic, and the paleontological evidence, which suggests ancestral mammals were small arboreal animals and most likely, easy prey $[50,51]$.

\section{More-recent photic adaptations in mammals}

The removal of the predation pressures imposed by the large dinosaurs during the Mesozoic (66 mya), left mammals with the opportunity to explore other photic environments $[38,42]$. We showed that three mammalians species (platypus, beard seal and Sowerby's beaked whale) have undergone more-recent adaptations for some of the studied opsins. The platypus lineage showed conserved adaptive evolution for the OPN4m and $R R H$ opsins, suggesting it has maintained the same circadian responses as the ancestral mammals. In agreement with this result, and considering the platypus possess several characteristics of a scotopic/mesopic-adapted eye (low visual acuity and a large optic tectum [52]), we suggest that platypus may be an efficient model-organism to study the ancestral mammalian photic system. The RH1 opsin showed accelerated evolution in the bearded seal and Sowerby's beaked whale lineages. The bearded seal and Sowerby's beaked whale species occupy the cold waters of the North Atlantic, where the low luminosity together with the necessity to dive in deep to feed, may have favoured the retuning of RH1. Fasick et al. (2000) and Zhao et al. (2009) showed that RH1 amino acid substitutions and spectral tuning shifts were correlated with foraging depth in marine mammals [22, 53]. Notice that an acceleration of the RH1 opsins was also found in the North Atlantic right whale (Eubalaena glacialis) lineage; however, it was not significant after the Bonferroni correction ( $p$-value $=0.0029 / 0.002$, Additional file 4: Table S3).

\section{Conclusions}

The genomic and phylogenetic lines of evidence advanced here provide new insights on the evolution of mammalian eyes: $(i)$ equal and differentiated patterns of opsin loss in mammals suggested a nocturnal period that affected both the common and the emerging lineages of mammals; in addition, we advance that ancestral mammals possessed (ii) a nocturnal activity pattern, (iii) UVS vision and a scotopic-adapted eye with (iv) low visual acuity and $(v)$ panoramic vision. In sum, we provide conclusive evidence that mammals (and not only eutherians as Walls initially assumed [1]) were scotopicadapted for most of their evolutionary history, supporting a global nocturnal bottleneck starting at $>>215.5$ mya and lasting until 66 mya.

\section{Methods}

\section{Synteny and phylogenetic analysis}

tblastn searches with protein sequences of the Homo sapiens and Gallus gallus opsins were performed in the NCBI and Ensembl databases [15, 16]. Synteny analyses were performed using both the Ensembl and Genomicus 64.1 databases $[16,54]$. Previously published sequences were collected representing the main phylogenetic groups of mammals for each of the studied opsins (Additional file 8: Table S5). A protein-based coding sequence alignment was performed using the Muscle 3.3 algorithm [55] and subsequently further alignment pruning was employed to remove ambiguous and/or gapsrich sites. The presence of saturation in base substitution for each of the opsin gene alignments was assessed via the Xia et al. (2003) test [56]; we found no evidence of saturation (Additional file 9: Table S6). jModelTest (version 0.1.1) with Akaike Information criterion was used to estimate the most appropriate nucleotide substitution model [57] and the GTR $+\mathrm{I}+\Gamma$ was the mostappropriate model with $95 \%$ of confidence for all opsins, with the exception of $R H 1$ gene, where $\mathrm{HKY}+\mathrm{I}+\Gamma$ was the best-fit model. 


\section{Site- and branch-selection analysis}

Site-specific and branch-specific codon substitution models of maximum likelihood, based on the $\omega$-statistic, were implemented in PAML [18]. To eliminate possible confounding effects of phylogeny variation and because we aimed to trace gene evolution within a framework of species evolution, the Meredith et al. (2011) mammalian species tree was employed [40]. The site-specific models were tested comparatively using the LRT: M7 (beta) vs. M8 (beta $+\omega)$ and M8a (beta $+\omega=1$ ) vs. M8 [17]. M7 and M8 assume a beta-distribution for the $\omega$ value between 0 and 1 but M8 additionally allows the occurrence of positively-selected sites $(\omega>1)$ [58]. M8a tests the neutral evolution including a class of neutral evolving sites [58]. Whenever the LRT was significant under the M8 model, the Bayes Empirical Bayes method was used to assess the positively-selected sites (accepted at $>0.75$ posterior probability) [59].

The branch selection models were carried out in two phases: computing the free-ratios model and testing the one-ratio vs. two-ratio hypothesis [21]. The free ratios model was implemented to calculate the opsin $\omega$-tree. The branches with an outlier $\omega$-ratio $(>>10$, mostly due to small $d S$ estimates) were recalculated as the ratio between the obtained $d N$ and the median $d S$. The speciesspecific evolutionary rates ( $\omega$-lineage), corresponding to the sum of the root-to-tip $\omega$-branches in each of the opsin $\omega$-trees (i.e. a $\omega$-tree linearization), were calculated and standardized to identify $\omega$-lineage outlier tendencies. The $\omega$-lineage was calculated using the distRoot(tree,tips) function of the adephylo package of the $\mathrm{R}$ statistical software $[60,61]$. Statistically significant $\omega$-lineage variations in specific branches were tested via LRT using the one-ratio vs. the two-ratio model comparison: the two-ratio model considers an additional $\omega$-branch parameter for the lineage of interest ( $\omega$-foreground) [21].

\section{Photic-related character dataset construction}

A dataset of four photic-related characters (Additional file 10: Table S7) was built for the studied mammalian species. Mammalian activity pattern was determined based on PanTHERIA database and Bennie et al. (2014) [62, 63]. Crepuscular and nocturnal activity patterns were both considered as nocturnal. We have used the amino acid composition at site 86 to perform the VS and UVS inferences. Site 86 has a major role in OPN1sw1 spectral tuning, since substitutions in this site have been shown to result in large absorption maxima shifts $[19,20]$. However, site 86 is not ubiquitous in the spectral tuning of the mammalian OPN1sw1and its absorption maximum can vary depending on the amino acid composition of other sites (namely 46, 49, 52, 81, 93, 114 and 118), as noted by Hauser et al. (2014) [64]. In order to avoid these caveats, we opted for using the VS/UVS discrete categories which are more stable for amino acid composition, instead of using absorption maxima measures: the 86F residue is associated with UVS (except in primates) while the $\mathrm{Y}, \mathrm{S}, \mathrm{V}, \mathrm{C}$ and $\mathrm{L}$ with VS (reviewed in $[5,11,64]$ ); other amino acid compositions were not considered to UVS/VS inference. In addition, mammalian species for which OPN1sw1 was reported inactive were excluded from the analysis (based on [65-71], Additional file 10: Table S7). Orbit convergence measurements for non-monotremes mammals were obtained in $[32,33]$. Orbit convergence measurements for the platypus and echidna monotremes were performed using the high-resolution computed tomography $\mathrm{x}$-ray skulls (Digimorph project [72], www.digimorph.org). Visual acuity data were obtained from Veilleux et al. (2014) [34]. Platypus and opossum visual acuity measures were retrieved from [52, 73].

\section{Ancestral character reconstruction analysis}

Ancestral character reconstructions were performed using the opsin $\omega$-trees and the data matrices in the ape package of the R statistical software [61, 74]: ace(data, tree) function with additional arguments. Discrete characters (type = "discrete") were calculated based on a time-continuous Markovian model comparing the equal-rates (model = "SYM") and all-rates-different (model = "ARD") matrices on a LRT test [75]. Ancestral states were estimated using maximum likelihood inference (if type = "discrete", maximum likelihood inferences are calculated by default). Continuous characters were firstly tested for phylogenetic autocorrelation with the opsin $\omega$-trees using the Moran's I statistic [76] that was calculated by the Moran.I() command. The significant associations ( $p$-value $<0.003$, Bonferroni corrected for 16 comparisons; Additional file 6: Table S4) were employed to perform inferences in ancestral nodes, using the maximum likelihood method (method = "ML") and the Brownian motion model (if type $=$ "continuous" is selected in the ace function, the default model is Brownian motion) [77].

\section{Additional files}

\begin{abstract}
Additional file 1: Table S1. Site-selection tests for the mammalian opsins. The logarithm of the model likelihood is represented by $\operatorname{lnL}$, the number of model parameters is represented by $\mathrm{np}$ and the LRT is the likelihood ratio test. The accepted site-selection model are indicated with an asterisk $\left(^{*}\right)$ when the M7 model of negative selection is statistically significant, or with a double asterisk (**) if the M8 model of positive selection is statically significant. All the LRT comparisons were performed assuming a significance level of 0.05. (PDF $273 \mathrm{~kb}$ )
\end{abstract}

Additional file 2: Table S2. Opsin $\omega$-tree maximum likelihood estimates. The $\omega$-tree was estimated under the assumption of the branch-specific free-ratios model using the Meredith et al. (2011) tree topology. (XLSX $17 \mathrm{~kb}$ )

Additional file 3: Figure S1. Species-specific evolutionary rate for mammalian opsins. $\omega$-lineages were standardized subtracting the median 
and divided by the interquartile range. Coloured circles correspond to the species subjected to branch selection tests and significant results are indicated with an asterisk (*). (PDF $501 \mathrm{~kb}$ )

Additional file 4: Table S3. Species-specific branch selection tests. The one-ratio model $(\mathrm{HO})$ was tested against the two-ratios model considering the alternative hypotheses $(\mathrm{H} 1)$ of verifying differentiated $\omega$-ratio in the indicated branch. InL is the logarithm of the model likelihood and the LRT is the likelihood ratio test. All the LRT comparisons were performed with 1 degree of freedom. Significant alternative hypothesis are marked with an asterisk (*) considering a Bonferroni corrected $p$-value of 0.002 (24 test comparisons). (PDF $278 \mathrm{~kb}$ )

Additional file 5: Figure S2. Phylogenetic character mapping for the ancestral reconstructions. Ancestral reconstructions of the activity pattern and SW1-sensitivity are represented in pie charts, each slice representing the probability of each state. Ancestral inferences of the orbit convergence (degrees) and visual acuity (cycles per degree) are represented by circles that change in size and shade of grey according to the character value. The opsin trees were estimated under the assumption of the branch-specific free-ratios model using the Meredith et al. (2011) tree topology. (PDF $1239 \mathrm{~kb})$

Additional file 6: Table S4. Ancestral character reconstructions of orbit convergence and visual acuity in mammals. The phylogenetic association between each variable and the opsin $\omega$-tree was tested using the Moran's I hypothesis test ( $p$-value $<0.003$, Bonferroni corrected for 16 comparisons). Ancestral character reconstructions were performed using the Brownian motion model, excluding the cases in which none $\left(^{*}\right)$ or only one $\left(^{* *}\right)$ species was representing the clades of interest (mammalia, monotremata, eutheria, marsupialia, placentalia). Inferences for each opsin (mean and standard deviation) are shown for each opsin gene tree. The last line of the table summarizes the results considering the mean probability distribution. (PDF $435 \mathrm{~kb}$ )

Additional file 7: Figure S3. Orbit convergence vs. activity patterns in mammals. Box plots depicting the association between the orbit convergence (degrees, ${ }^{\circ}$ ) and the activity pattern (nocturnal and diurnal) of extant mammals. (PDF $276 \mathrm{~kb}$ )

Additional file 8: Table S5. Mammalian opsin sequences. Accession number of the mammalian opsins sequences used for the evolutionary analyses. (XLSX $103 \mathrm{~kb}$ )

Additional file 9: Table S6. Saturation analysis. The presence of saturation in base substitution for each of the opsin gene alignment was tested by comparing half of the expected theoretical saturation index when assuming full saturation (Iss.c, critical value) with the observed saturation index (ISS). The absence of substitution saturation is verified when Iss is lower than Iss.c for a significant p-value. (PDF $107 \mathrm{~kb}$ )

Additional file 10: Table S7. Dataset of the eco-morphological variables for the studied mammalian species. Variables: activity pattern (nocturnal, cathemeral and diurnal), VS and UVS OPN1sw1 sensitivity, orbit convergence (degrees, ${ }^{\circ}$ ) and visual acuity (cycle per degree, cpd). OPN1sw1 inactive copies were indicated with an asterisk $\left({ }^{*}\right)$ in the column of the sw1 86 site. Data retrieved from the references [5, 11, 65-72]. (XLSX $22 \mathrm{~kb})$

\section{Acknowledgements}

We thank João Paulo Machado for discussion and helpful suggestions.

\section{Funding}

$\mathrm{RB}$ and CG were funded with a PhD grant from Fundação para a Ciência e a Tecnologia (FCT) (RB: SFRH/BD/79850/2011 and CG: SFRH/BD/71041/2010). SJO was supported, in part, by the Russian Science Foundation grant (project No. 17-14-01138) and by St. Petersburg State University (Genome Russia Grant no. 1.52.1647.2016). AA was partially supported by the Strategic Funding UID/Multi/04423/2013 through national funds provided by FCT and the European Regional Development Fund (ERDF) in the framework of the program PT2020, by the European Structural and Investment Funds (ESIF) through the Competitiveness and Internationalization Operational Program COMPETE 2020 and by National Funds through the FCT under the projects PTDC/AAG-GLO/6887/2014 (POCI-01-0124-FEDER-016845).

\section{Availability of data and materials}

The datasets generated and/or analysed during the current study are available in the supplementary material.

\section{Authors' contributions}

RB performed the phylogenetic, evolutionary and ancestral state reconstruction analyses and drafted the manuscript. WEJ and SJO participated in the drafting and coordination of the study. CG performed the initial gene mining analysis and ancestral reconstruction analysis. CPH contributed with data and participated in the drafting of the manuscript. AA participated in the design, genetic analyses, drafting and coordination of the study. All authors read and approved the final manuscript.

\section{Ethics approval and consent to participate}

Not applicable.

\section{Consent for publication}

Not applicable.

\section{Competing interests}

The authors declare they have no competing interests.

\section{Publisher's Note}

Springer Nature remains neutral with regard to jurisdictional claims in published maps and institutional affiliations.

\section{Author details}

${ }^{1}$ CIIMAR/CIMAR, Interdisciplinary Centre of Marine and Environmental Research, University of Porto, Terminal de Cruzeiros do Porto de Leixões, Av. General Norton de Matos, s/n, 4450-208, Porto, Portugal. ${ }^{2}$ Department of Biology, Faculty of Sciences, University of Porto, Rua do Campo Alegre, 4169-007 Porto, Portugal. ${ }^{3}$ Smithsonian Conservation Biology Institute, National Zoological Park, 1500 Remount Road, Front Royal, VA 22630, USA. ${ }^{4}$ Theodosius Dobzhansky Center for Genome Bioinformatics, St. Petersburg State University, St. Petersburg, Russia199004. ${ }^{5}$ Guy Harvey Oceanographic Center, Halmos College of Natural Sciences and Oceanography, Nova Southeastern University, 8000, North Ocean Drive, Ft Lauderdale 33004, Florida, USA. ${ }^{6}$ ICBAS, Institute of the Biomedical Sciences of Abel Salazar, University of Porto, Rua de Jorge Viterbo Ferreira, 228, 4050-313 Porto, Portugal. ${ }^{7}$ Department of Anatomy, Arizona College of Osteopathic Medicine, Midwestern University, 19555 N. 59th avenue, Glendale, AZ, USA.

Received: 30 November 2016 Accepted: 22 December 2017 Published online: 05 February 2018

\section{References}

1. Walls G. The vertebrate eye and its adaptive radiation. New York: Hafner publishing company; 1942

2. Ahnelt PK, Kolb H. The mammalian photoreceptor mosaic-adaptive design. Prog Retin Eye Res. 2000;19:711-77.

3. Arendt J. Melatonin and the pineal gland: influence on mammalian seasonal and circadian physiology. Rev Reprod. 1998;3:13-22.

4. Peirson S, Halford S, Foster R. The evolution of irradiance detection: melanopsin and the non-visual opsins. Philos Trans R Soc Lond Ser B Biol Sci. 2009;364:2849-65.

5. Hunt DM, Carvalho LS, Cowing JA, Davies WL. Evolution and spectral tuning of visual pigments in birds and mammals. Philos Trans R Soc Lond Ser B Biol Sci. 2009;364:2941-55.

6. Bowmaker JK. Evolution of vertebrate visual pigments. Vision Res. [Internet]. 2008;48:2022-41. Available from: http://linkinghub.elsevier.com/retrieve/pii/ S004269890800148X.

7. Hall MI, Kamilar JM, Kirk EC. Eye shape and the nocturnal bottleneck of mammals. Proc R Soc B Biol Sci [Internet]. 2012;279:4962-8. Available from: http://rspb.royalsocietypublishing.org/cgi/doi/10.1098/rspb.2012.2258.

8. Heesy CP, Hall MI. The nocturnal bottleneck and the evolution of mammalian vision. Brain Behav Evol. 2010;75:195-203.

9. Heesy CP. Ecomorphology of orbit orientation and the adaptive significance of binocular vision in primates and other mammals. Brain Behav Evol. 2008;71:54-67. 
10. Howland HC, Merola S, Basarab JR. The allometry and scaling of the size of vertebrate eyes. Vision Res [Internet]. 2004;44:2043-65. Available from: http://linkinghub.elsevier.com/retrieve/pii/S0042698904001646.

11. Bowmaker JK, Hunt DM. Evolution of vertebrate visual pigments. Curr Biol. 2006;16:R484-9.

12. Yokoyama S. Molecular evolution of vertebrate visual pigments. Prog Retin Eye Res. 2000;19:385-419.

13. Borges R, Johnson WE, O'Brien SJ, Vasconcelos V, Antunes A. The role of gene duplication and unconstrained selective pressures in the melanopsin gene family evolution and vertebrate circadian rhythm regulation. PLoS One. 2012;7:e52413.

14. Bellingham J, Chaurasia SS, Melyan Z, Liu C, Cameron MA, Tarttelin EE, et al. Evolution of melanopsin photoreceptors: discovery and characterization of a new melanopsin in nonmammalian vertebrates. PLoS Biol. 2006;4:e254.

15. McEntyre J, Ostell J, Pruitt K, Brown G. The reference sequence (RefSeq) database. In: McEntyre JOJ, editor. The NCBI H. 2nd ed; 2012. [cited 2014 Aug 19];http://www.ncbi.nlm.nih.gov/books/NBK143764/. Available from: http://www.ncbi.nlm.nih.gov/books/NBK21101/.

16. Cunningham F, Amode MR, Barrell D, Beal K, Billis K, Brent $S$, et al. Ensembl 2015. Nucleic Acids Res. 2015;43:D662-9. Available from: http://nar. oxfordjournals.org/lookup/doi/10.1093/nar/gku1010.

17. Anisimova M, Bielawski JP, Yang Z. Accuracy and power of the likelihood ratio test in detecting adaptive molecular evolution. Mol Biol Evol. 2001;18:1585-92.

18. Yang Z. PAML 4: Phylogenetic analysis by maximum likelihood. Mol Biol Evol. 2007;24:1586-91. Available from: http://mbe.oxfordjournals.org/cgi/doi/ 10.1093/molbev/msm088.

19. Carvalho LS, Davies WL, Robinson PR, Hunt DM. Spectral tuning and evolution of primate short-wavelength-sensitive visual pigments. Proc R Soc B Biol Sci. 2012;279:387-93. Available from: http://rspb. royalsocietypublishing.org/cgi/doi/10.1098/rspb.2011.0782.

20. Shi Y, Radlwimmer FB, Yokoyama S. Molecular genetics and the evolution of ultraviolet vision in vertebrates. Proc Natl Acad Sci. 2001;98:11731-6. Available from: http://www.pnas.org/cgi/doi/10.1073/pnas.201257398.

21. Yang Z, Nielsen R. Codon-substitution models for detecting molecular adaptation at individual sites along specific lineages. Mol Biol Evol. 2002;19:908-17.

22. Zhao H, Ru B, Teeling E, Faulkes C, Zhang S, Rossiter S. Rhodopsin molecular evolution in mammals inhabiting low light environments. PLoS One [Internet]. 2009:4:e8326. Scheffler K, editor. Available from: http://dx.plos.org/ 10.1371/journal.pone.0008326.

23. Davies WIL, Collin SP, Hunt DM. Molecular ecology and adaptation of visual photopigments in craniates. Mol Ecol. 2012;21:3121-58. Available from: http://doi.wiley.com/10.1111/j.1365-294X.2012.05617.x.

24. Bickelmann C, Morrow JM, Du J, Schott RK, van Hazel I, Lim S, et al. The molecular origin and evolution of dim-light vision in mammals. Evolution (N Y). 2015;69:2995-3003. Available from: http://doi.wiley.com/ 10.1111/evo.12794.

25. Fernandez-Sampedro MA, Invergo BM, Ramon E, Bertranpetit J, Garriga P. Functional role of positively selected amino acid substitutions in mammalian rhodopsin evolution. Sci Rep. 2016;6:21570. Available from: http://www.nature.com/articles/srep21570.

26. Yokoyama S, Tada T, Zhang H, Britt L. Elucidation of phenotypic adaptations: molecular analyses of dim-light vision proteins in vertebrates. Proc Natl Acad Sci. 2008;105:13480-5. Available from: http://www.pnas.org/cgi/doi/10. 1073/pnas.0802426105

27. Cowing JA, Poopalasundaram S, Wilkie SE, Robinson PR, Bowmaker JK, Hunt DM. The molecular mechanism for the spectral shifts between vertebrate ultraviolet- and violet-sensitive cone visual pigments. Biochem J. 2002;367: 129-35. [cited 2014 Dec 11] Available from: http://www.pubmedcentral.nih. gov/articlerender.fcgi?artid=1222874\&tool=pmcentrez\&rendertype=abstract.

28. Fasick Jl, Applebury ML, Oprian DD. Spectral tuning in the mammalian short-wavelength sensitive cone pigments †. Biochemistry. 2002;41:6860-5. [cited 2014 Dec 11] Available from: http://pubs.acs.org/doi/abs/10.1021/ bi0200413.

29. Yokoyama S, Shi Y, Yokoyama S. Genetics and evolution of ultraviolet vision in vertebrates. FEBS Lett. 2000;486:167-72. [cited 2014 Dec 11]; Available from: http://linkinghub.elsevier.com/retrieve/pii/S0014579300022699.

30. Hunt DM, Carvalho LS, Cowing J, Parry JWL, Wilkie SE, Davies WL, et al. Spectral tuning of shortwave-sensitive visual pigments in vertebrates Photochem. Photo-Dermatology. 2007;83:303-10.
31. Wheeler BC, Bradley BJ, Kamilar JM. Predictors of orbital convergence in primates: a test of the snake detection hypothesis of primate evolution. J Hum Evol. 2011;61:233-42. Elsevier Ltd

32. Heesy CP. On the relationship between orbit orientation and binocular visual field overlap in mammals. Anat Rec. 2004;281A:1104-10. Available from: http://doi.wiley.com/10.1002/ar.a.20116.

33. Heesy CP, Kamilar JM, Willms J. Retinogeniculostriate pathway components scale with orbit convergence only in primates and not in other mammals. Brain Behav Evol. 2011;77:105-15. Available from: http://www.karger.com/ doi/10.1159/000324860.

34. Veilleux CC, Kirk EC. Visual acuity in mammals: effects of eye size and ecology. Brain Behav Evol. 2014;83:43-53. Available from: http://www.karger. com?doi=10.1159/000357830.

35. Borges R, Khan I, Johnson WEWE, Gilbert MTPTP, Zhang G, Jarvis EDED, et al Gene loss, adaptive evolution and the co-evolution of plumage coloration genes with opsins in birds. BMC Genomics. 2015;16:1-13. Available from: https://doi.org/10.1186/s12864-015-1924-3

36. Vígh B, Manzano MJ, Zádori A, Frank CL, Lukáts $A$, Röhlich $P$, et al. Nonvisual photoreceptors of the deep brain, pineal organs and retina. Histol Histopathol. 2002;17:555-90.

37. Kemp TS. The origin of mammalian endothermy: a paradigm for the evolution of complex biological structure. Zool J Linnean Soc. 2006;147:473-88.

38. Gerkema MP, Davies WIL, Foster RG, Menaker M, Hut RA. The nocturnal bottleneck and the evolution of activity patterns in mammals. Proc Biol Sci. 2013;280:20130508. [cited 2014 Mar 27]; Available from: http://www.ncbi. nlm.nih.gov/pubmed/23825205.

39. Ralph CL, Firth BT, Turner JS. The role of the pineal body in Ectotherm thermoregulation. Am Zool. 1979;19:273-93. Available from: https:// academic.oup.com/icb/article-lookup/doi/10.1093/icb/19.1.273.

40. Meredith RW, Janečka JE, Gatesy J, Ryder OA, Fisher CA, Teeling EC, et al. Impacts of the cretaceous terrestrial revolution and KPg extinction on mammal diversification. Science. 2011;334:521-4. Available from: http:// www.ncbi.nlm.nih.gov/pubmed/21940861.

41. Angielczyk KD, Schmitz L. Nocturnality in synapsids predates the origin of mammals by over 100 million years. Proc R Soc B Biol Sci. 2014;281: 20141642. Available from: http://rspb.royalsocietypublishing.org/cgi/doi/10. 1098/rspb.2014.1642

42. Smith FA, Boyer AG, Brown JH, Costa DP, Dayan T, Ernest SKM, et al. The evolution of maximum body size of terrestrial mammals. Science. 2010;330:1216-9.

43. Liu J, Liu MY, Nguyen JB, Bhagat A, Mooney V, Yan ECY. Thermal properties of rhodopsin: insight into the molecular mechanism of dim-lingth vision. J Biol Chem. 2011;286:27622-9. Available from: http://www.jbc.org/cgi/doi/10. 1074/jbc.M111.233312.

44. Kim J-W, Yang H-J, Oel AP, Brooks MJ, Jia L, Plachetzki DC, et al. Recruitment of rod photoreceptors from short-wavelength-sensitive cones during the evolution of nocturnal vision in mammals. Dev Cell. 2016;37:520-32. Available from: http://linkinghub.elsevier.com/retrieve/pii/S1534580716303367.

45. Zele AJ, Cao D. Vision under mesopic and scotopic illumination. Front Psychol. 2015;5 Available from: http://journal.frontiersin.org/article/10.3389/ fpsyg.2014.01594/abstract.

46. Hughes A. The topography of vision in mammals of contrasting life style: comparative optics and retinal organisation. Vis Syst Vertebr. 1977:613-756. Springer Berlin Heidelberg; Available from: http://www.springerlink.com/ index/10.1007/978-3-642-66468-7_11.

47. Wikler KC, Rakic P. Distribution of photoreceptor types in the retina of diurnal and nocturnal primates. J Neurosci. 1990;10:3390-401.

48. Fitzpatrick D. Vision: the eye. In: Neuroscience 3rd edition, editor: Purves D, Augustine GJ, Fitzpatrick D, Hall WC, Lamantia A.-S, Mcnamara JO, Willians SM. Sunderland; 2004. p. 240-4

49. Orlowski J, Harmening W, Wagner H. Night vision in barn owls: visual acuity and contrast sensitivity under dark adaptation. J Vis. 2012;12:4. [cited 2014 Mar 27]; Available from: http://www.ncbi.nlm.nih.gov/pubmed/23220576.

50. Rowe TB, Macrini TE, Luo Z-X. Fossil evidence on origin of the mammalian brain. Science. 2011; [cited 2013 Aug 12];332:955-7. Available from: http:// www.ncbi.nlm.nih.gov/pubmed/21596988.

51. Luo ZX, Crompton AW, Sun AL. A new mammaliaform from the early Jurassic and evolution of mammalian characteristics. Science. 2001;292: 1535-40. Available from: http://www.ncbi.n/m.nih.gov/pubmed/11375489.

52. Pettigrew JD, Manger PR, Fine SLB. The sensory world of the platypus. Philos Trans R Soc B Biol Sci. 1998;353:1199-210. Available from: http://rstb. royalsocietypublishing.org/cgi/doi/10.1098/rstb.1998.0276. 
53. Fasick Jl, Robinson PR. Spectral-tuning mechanisms of marine mammal rhodopsins and correlations with foraging depth. Vis Neurosci. 2000;17: S095252380017511X. Available from: http://www.journals.cambridge.org/ abstract_S095252380017511X.

54. Muffato M, Louis A, Poisnel C-E, Roest Crollius H. Genomicus: a database and a browser to study gene synteny in modern and ancestral genomes. Bioinformatics. 2010;26:1119-21.

55. Edgar RC. MUSCLE: multiple sequence alignment with high accuracy and high throughput. Nucleic Acids Res. 2004;32:1792-7. Available from: https:// academic.oup.com/nar/article-lookup/doi/10.1093/nar/gkh340.

56. Xia X, Xie Z, Salemi M, Chen L, Wang Y. An index of substitution saturation and its application. Mol Phylogenet Evol. 2003;26:1-7.

57. Posada D. jModelTest: phylogenetic model averaging. Mol Biol Evol. 2008;25:1253-6.

58. Wong WSW. Accuracy and power of statistical methods for detecting adaptive evolution in protein coding sequences and for identifying positively selected sites. Genetics. 2004;168:1041-51. Available from: http:// www.genetics.org/cgi/doi/10.1534/genetics.104.031153.

59. Yang Z, Wong WSW, Nielsen R. Bayes empirical bayes inference of amino acid sites under positive selection. Mol Biol Evol. 2005;22:1107-18.

60. Pavoine S, Ollier S, Pontier D, Chessel D. Testing for phylogenetic signal in phenotypic traits: new matrices of phylogenetic proximities. Theor Popul Biol. 2008;73:79-91. Available from: http://linkinghub.elsevier.com/retrieve/ pii/S0040580907001177.

61. R Development Core Team R. R: a language and environment for statistical computing [internet]. Vienna: R Foundation for Statistical Computing; 2014. [cited 2016 Mar 15] Available from: http://www.r-project.org.

62. Jones KE, Bielby J, Cardillo M, Fritz SA, O'Dell J, Orme CDL, et al. PanTHERIA: a species-level database of life history, ecology, and geography of extant and recently extinct mammals. Ecology. 2009;90:2648. Michener WK, editor. Available from: http://www.esajournals.org/doi/abs/10.1890/08-1494.1.

63. Bennie JJ, Duffy JP, Inger R, Gaston KJ. Biogeography of time partitioning in mammals. Proc Natl Acad Sci. 2014;111:13727-32. Available from: http:// www.pnas.org/cgi/doi/10.1073/pnas.1216063110.

64. Hauser FE, van Hazel I, Chang BSW. Spectral tuning in vertebrate short wavelength-sensitive 1 (SWS1) visual pigments: can wavelength sensitivity be inferred from sequence data? J Exp Zool Part B Mol Dev Evol. 2014;322: 529-39. Available from: http://doi.wiley.com/10.1002/jez.1855.

65. Griebel U, Peichl L. Colour vision in aquatic mammals - facts and open questions. Aquat Mamm [Internet]. 2003;29:18-30. Available from: http:// www.aquaticmammalsjournal.org/index.php?option=com_content\&view= article\&id=208:-colour-vision-in-aquatic-mammalsfacts-and-open-questions\&catid=12:volume-29-issue-1\&ltemid=92.

66. Bischoff N, Nickle B, Cronin TW, Velasquez S, Fasick Jl. Deep-sea and pelagic rod visual pigments identified in the mysticete whales. Vis Neurosci. 2012; 29:95-103. Available from: http://www.journals.cambridge.org/abstract_ S0952523812000107.

67. Meredith RW, Gatesy J, Emerling CA, York VM, Springer MS. Rod Monochromacy and the Coevolution of cetacean retinal Opsins. PLoS Genet. 2013;9:e1003432. Zhang J, editor. Available from: http://dx.plos.org/ 10.1371/journal.pgen.1003432.

68. Emerling CA, Springer MS. Eyes underground: regression of visual protein networks in subterranean mammals. Mol Phylogenet Evol. 2014;78:260-70. Available from: http://linkinghub.elsevier.com/retrieve/pii/S105579031400181X.

69. Emerling CA, Springer MS. Genomic evidence for rod monochromacy in sloths and armadillos suggests early subterranean history for Xenarthra. Proc R Soc B Biol Sci. 2014;282:20142192. Available from: http://rspb. royalsocietypublishing.org/cgi/doi/10.1098/rspb.2014.2192.

70. Emerling CA, Huynh HT, Nguyen MA, Meredith RW, Springer MS. Spectral shifts of mammalian ultraviolet-sensitive pigments (short wavelengthsensitive opsin 1) are associated with eye length and photic niche evolution. Proc R Soc B Biol Sci. 2015;282:20151817. Available from: http:// rspb.royalsocietypublishing.org/lookup/doi/10.1098/rspb.2015.1817.

71. Springer MS, Emerling CA, Fugate N, Patel R, Starrett J, Morin PA, et al. Inactivation of cone-specific Phototransduction genes in rod monochromatic cetaceans. Front Ecol Evol. 2016;4 Available from: http:// journal.frontiersin.org/Article/10.3389/fevo.2016.00061/abstract.

72. Rowe T, Humphries J, Maisano J, Ketcham R, Colbert M. DigiMorph. 2015 [cited 2015 Jan 12]. Available from: http://www.digimorph.org/.

73. Dooley JC, Nguyen HM, Seelke AMH, Krubitzer L. Visual acuity in the shorttailed opossum (Monodelphis Domestica). Neuroscience. 2012;223:124-30.
Available from: http://linkinghub.elsevier.com/retrieve/pii/ S0306452212008135.

74. Paradis E, Claude J, Strimmer K. APE: analyses of Phylogenetics and evolution in R language. Bioinformatics. 2004;20:289-90. Available from: http:// bioinformatics.oxfordjournals.org/cgi/doi/10.1093/bioinformatics/btg412.

75. Pagel M. Detecting correlated evolution on phylogenies: a general method for the comparative analysis of discrete characters. Proc R Soc B Biol Sci. 1994;255:37-45. Available from: http://rspb.royalsocietypublishing.org/cgi/ doi/10.1098/rspb.1994.0006.

76. Gittleman JL, Kot M. Adaptation: statistics and a null model for estimating Phylogenetic effects. Syst Zool. 1990;39:227. Available from: http://sysbio. oxfordjournals.org/cgi/doi/10.2307/2992183.

77. Schluter D, Price T, Mooers AO, Ludwig D. Likelihood of ancestor states in adaptive radiation. Evolution (N. Y). 1997;51:1699. Available from: http:// www.jstor.org/stable/2410994?origin=crossref

78. Terakita A. The opsins. Genome Biol. 2005:1-9.

\section{Submit your next manuscript to BioMed Central and we will help you at every step:}

- We accept pre-submission inquiries

- Our selector tool helps you to find the most relevant journal

- We provide round the clock customer support

- Convenient online submission

- Thorough peer review

- Inclusion in PubMed and all major indexing services

- Maximum visibility for your research

Submit your manuscript at www.biomedcentral.com/submit
C Biomed Central 